\title{
Analysis of Genetic Variation of VP2 Gene in 6 FPV Strainsin China
}

\author{
Jianxin Wen*, Zhiqiang Wang, Jianwei Ren \\ College of Veterinary Medicine, Qingdao Agricultural University, Qingdao, China \\ Email: *wenjianxin@126.com
}

How to cite this paper: Wen, J.X., Wang, Z.Q. and Ren, J.W. (2021) Analysis of Genetic Variation of VP2 Gene in 6 FPV Strainsin China. Advances in Microbiology, 11, 191-198.

https://doi.org/10.4236/aim.2021.114014

Received: March 14, 2021

Accepted: April 22, 2021

Published: April 25, 2021

Copyright $\odot 2021$ by author(s) and Scientific Research Publishing Inc. This work is licensed under the Creative Commons Attribution International License (CC BY 4.0).

http://creativecommons.org/licenses/by/4.0/

(c) (i) Open Access

\begin{abstract}
In order to understand the variation of FPV strains in the Jinan area, Shandong Province, China, the VP2 gene of 6 FPV strains was sequenced, and the analysis of the genetic relationship, evolution and main functional site variation was carried out. It was found that FPV-XY2, FPV-XY3 and FPV-XY6 were the same strain with $100 \%$ homology, and also close to FPV-XY1, and the homology between FPV-XY4 and FPV-XY5 was close. The homology between the reference strain and the test strain was over $99.3 \%$. According to the evolutionary analysis, the genetic relationship among FPV-XY1, FPV-XY2, FPV-XY3, FPV-XY6 was close, and the genetic relationship between FPV-XY4 and FPV-XY5 was close, and the result was similar to the homologous result. Compared with the VP2 amino acid sequence of the standard strain FPV-CU4, the VP2 protein of all the test strains changed from I to $t$ on the 101st Amino acid, this may be the cause of immune failure in these 6 cases; the change of a to $s$ in the 91st amino acid position of FPV-XY1, FPV-XY2, FPV-XY3, FPV-XY6 may be the cause of enhanced virulence of FPV. This study provides a reference for exploring the epidemic law of FP in the Jinan area, the standard of FP treatment plan and the research and development of FPV subunit vaccine.
\end{abstract}

\section{Keywords}

Feline Panleukopenia, Feline Panleukopenia Virus, VP2 Gene, Evolutionary

\section{Analysis}

FPV belongs to the genus Parvovirus, which is currently distributed all over the world. Under natural conditions, it can infect carnivorous animals such as cats, leopards, tigers, and lions. FPV is closely related to MEV and CPV. Studies have shown that their nucleotide sequence similarity can reach more than $98 \%$. There are differences between them mainly in certain nucleocapsid proteins. Their cell 
culture in vitro is very similar to [1]. FPV is a single-stranded linear DNA virus with a diameter of about $25 \mathrm{~nm}$ and a full gene length of about $5000 \mathrm{bp}$. FPV currently reports only one serotype. FPV is mainly composed of two structural proteins and two non-structural proteins. The two structural proteins are VP1 and VP2, and the two non-structural proteins are NS1 and NS2. The VP1 protein is composed of 727 amino acids, and the VP2 protein is composed of 584 amino acids. The VP2 sequence is completely contained in the VP1 sequence [2]. There are $5 \mathrm{key}$ amino acid positions in the VP2 protein, namely the 80th, 103rd, 323, 504, and 568 positions. They are related to the antigenicity, hemagglutination, and host specificity of the virus. The neutralizing antigen site of FPV is also located on the VP2 protein, which can well induce the body to produce antibodies, cause an immune response, and can also be used for preparing vaccines. The FPV-CU4 strain was first reported by Parrish, CR in the United States in 1990, and was later used as a standard strain in vaccine production and antibody production [3]. At present, it has been clinically found that some cats are still infected by FPV even after receiving the vaccine, and some cats have died. There are opinions that there may be mutations in the current epidemic strains in Jinan. In order to understand the mutation of FPV in the Jinan area, Shandong Province, China, the study selected 6 cases of cats, all of which were received by a chain animal hospital in Jinan who were still infected with FPV after immunization and were treated and nursed by me. Among them, there were 3 dead cases. Stool samples were collected from them and their DNA was extracted. Use the PCR method to sequence its VP2 gene, select reference sequence and standard strain sequence for genetic analysis and genetic evolution analysis, and perform mutation analysis with reference to the nucleotide sequence of standard strain. This study provides a theoretical basis for the prevention and control of FPV in Jinan, and provides a reference for the development of FPV subunit vaccines.

\section{Material}

1) Samples among the 24 cases vaccinated with FPV, 6 cases were still infected with FPV after being immunized with the vaccine, and 3 cases died after treatment. As shown in Table 1 Stool samples were taken from these 6 cases and stored in a refrigerator at $-20^{\circ} \mathrm{C}$.

2) Reagents:

The viral DNA extraction kit was purchased from Tiangen Biotechnology, agarose was purchased from Haibo Biotechnology Co., Ltd., DNA Marker DL2000 was purchased from Takara Company, Premix was purchased from Takara Company, and nucleic acid dye was purchased from Beijing Soleibao Company.

3) Main instruments and equipment:

Biological safety cabinet (SW-CJ-2F, Sujing Group Antai Company); digital display constant temperature water bath (HWS-26, Shanghai Yiheng Company); desktop centrifuge (MiniSpin, Germany Aibende Company); Adjustable micro- 
pipette (Germany Eppendorf company); PCR machine (Takara company); gel imaging system (Beijing Kechuangrui New Biotechnology Co., Ltd.); electrophoresis tank (JY-SCZ2 + type, Beijing Junyi Dongfang Electrophoresis Equipment Co., Ltd.).

\section{Method}

1) Virus DNA extraction According to the instructions of the TIANamp Virus DNA/RNA Kit, extract the virus DNA from the 6 case samples and store them in a refrigerator at $-20^{\circ} \mathrm{C}$ for later use.

2) Primer design and synthesis according to the literature [2], the specific primers for the VP2 gene of FPV are synthesized:

FPV-VP2-F 5'-3': GCTGCTTATCTTCGCTC

FPV-VP2-R 5'-3': AACAAACCTTCTAAATCCTAT

3) VP2 gene amplification:

Use the extracted viral DNA as a template to establish a $25 \mu \mathrm{L}$ PCR reaction system, and set a negative control without template. The reaction system is as follows: template $0.5 \mu \mathrm{L}$, upstream and downstream primers $1 \mu \mathrm{L}$ each, Premix $12.5 \mu \mathrm{L}, \mathrm{ddH}_{2} \mathrm{O} 10 \mu \mathrm{L}$. PCR reaction conditions: pre-denaturation at $95^{\circ} \mathrm{C}$ for 5 min, start cycle: denaturation at $94^{\circ} \mathrm{C}$ for $1 \mathrm{~min}$, annealing at $51^{\circ} \mathrm{C}$ for $1 \mathrm{~min}$, extension at $72^{\circ} \mathrm{C}$ for $2 \mathrm{~min} 30 \mathrm{~s}, 30$ cycles, and finally at $72^{\circ} \mathrm{C}$ for $10 \mathrm{~min} .1 .0 \%$ agarose gel electrophoresis to check the PCR products. Send the amplified PCR products to Shanghai Parsons Biotechnology Co., Ltd. for sequencing.

4) VP2 gene homology analysis:

Firstly use NCBI (https://www.ncbi.nlm.nih.gov/) to perform BLAST comparison of the gene sequence obtained by sequencing, and select the first 4 sequences of each gene sequence comparison result to download, As FPV reference sequence. After sorting it out, it was found that there were multiple reference sequences with duplication. After removing the repetitive sequences, 7 were selected as the reference sequence (Table 2), including the standard sequence FPV-CU4 strain, and use MegAlign software to perform homology analysis on the sequenced sequences. Source analysis.

Table 1. Stool sample pet information.

\begin{tabular}{|c|c|c|c|c|c|}
\hline Sample serial number & Breed & Age & Gender & Clinical symptoms & Situation \\
\hline 1 & Chinese Garden Cat & 6 months old & female & Severe diarrhea & death \\
\hline 2 & British Shorthair & 3 months old & female & Vomiting, diarrhea & death \\
\hline 3 & doll cat & 2 months old & male & Severe diarrhea & death \\
\hline 4 & American Shorthair & 4 months old & female & Diarrhea & cure \\
\hline 5 & doll cat & 3 months old & female & Vomiting, diarrhea & cure \\
\hline 6 & Chinese Garden Cat & 1 year old & male & Diarrhea & cure \\
\hline
\end{tabular}


Table 2. Reference sequence.

\begin{tabular}{ccccc}
\hline Serial number & Virus strain & Login ID & $\begin{array}{c}\text { Submission } \\
\text { date }\end{array}$ & $\begin{array}{c}\text { Country } \\
\text { of origin }\end{array}$ \\
\hline 1 & FPV-GB & AY665655.1 & 2016 & Russia \\
2 & FPV-IZSSI & KX434461.1 & 2019 & Italy \\
3 & FPV-HH6 & KX900570.1 & 2017 & China \\
4 & FPV-CU4 & M38246.1 & 1990 & USA \\
5 & FPV-M9132 & KP769859.1 & 2016 & Belgium \\
6 & FPV-Raccoon & MF069445.1 & 2017 & Canada \\
7 & FPV-G & MG764510.1 & 2018 & China \\
\hline
\end{tabular}

5) Construction of VP2 gene:

Phylogenetic tree selected 7 reference strain reference sequences and the 6 test strain sequences obtained by sequencing were used to draw the VP2 gene phylogenetic tree using MEGA 7.0 software and Neighbor-Joining method (default parameters).

6) Analysis of the main amino acid site variation of the VP2 gene:

The 6 strains of VP2 genome sequences obtained from the sequencing were processed for protein translation with Bioedit software, and the processed amino acid sequence was compared with the VP2 amino acid sequence of the standard strain FPV-CU4 Comparison and analysis of main amino acid positions.

\section{Results}

1) Results of PCR product gel electrophoresis:

The PCR product was subjected to $1 \%$ agarose gel electrophoresis, and it was found that the amplified fragments of the 6 PCR products were all consistent with the expected amplified fragment size. The size of the target fragment was about 2200 bp, which were named respectively FPV-XY1, FPV-XY2, FPV-XY3, FPV-XY4, FPV-XY5 and FPV-XY6.

2) Homology analysis of VP2 gene Comparing the nucleotide sequence of the VP2 gene of the 6 FPV strains obtained by sequencing with the VP2 reference sequence of the selected FPV, it is found (see Figure 1) that the homology between FPV-XY1 and FPV-XY6 is $99.4 \%-100 \%$, the homology of the three samples FPV-XY2, FPV-XY3, and FPV-XY6 is $100 \%$, which is determined to be the same strain; the homology between the test sample and the reference sample is $99.3 \%$ - 99.7\%. Between them, indicating higher homology and smaller differences. In addition, in comparison, FPV-XY1, FPV-XY2, FPV-XY3, and FPV-XY6 are more similar, and FPV-XY4 and FPV-XY5 are more similar; reference strains FPV-HH6 and FPV-IZSSI. It is more similar to the test strain, and the difference is small. The homology between the test strain and the standard strain FPV-CU4 is between $99.3 \%-99.5 \%$, which is quite different in comparison. 


\begin{tabular}{|c|c|c|c|c|c|c|c|c|c|c|c|c|c|c|}
\hline \multicolumn{15}{|c|}{ Percent Identity } \\
\hline & 1 & 2 & 3 & 4 & 5 & 6 & 7 & 8 & 9 & 10 & 11 & 12 & 13 & \\
\hline 1 & & 99.9 & 99.9 & 99.4 & 99.4 & 99.9 & 99.5 & 99.3 & 99.5 & 99.6 & 99.6 & 99.4 & 99.5 & 1 \\
\hline 2 & 0.0 & & 100.0 & 99.4 & 99.4 & 100.0 & 99.5 & 99.4 & 99.6 & 99.7 & 99.7 & 99.5 & 99.6 & 2 \\
\hline 3 & 0.0 & 0.0 & & 99.4 & 99.4 & 100.0 & 99.5 & 99.4 & 99.6 & 99.7 & 99.7 & 99.5 & 99.6 & 3 \\
\hline 4 & 0.6 & 0.6 & 0.6 & & 99.8 & 99.4 & 99.3 & 99.4 & 99.5 & 99.7 & 99.5 & 99.4 & 99.4 & 4 \\
\hline 5 & 0.6 & 0.6 & 0.6 & 0.2 & & 99.4 & 99.3 & 99.5 & 99.6 & 99.7 & 99.7 & 99.4 & 99.4 & 5 \\
\hline 6 & 0.0 & 0.0 & 0.0 & 0.6 & 0.6 & & 99.5 & 99.4 & 99.6 & 99.7 & 99.7 & 99.5 & 99.6 & 6 \\
\hline 7 & 0.5 & 0.5 & 0.5 & 0.7 & 0.7 & 0.5 & & 99.3 & 99.6 & 99.5 & 99.5 & 99.4 & 99.8 & 7 \\
\hline 8 & 0.6 & 0.6 & 0.6 & 0.6 & 0.5 & 0.6 & 0.7 & & 99.5 & 99.7 & 99.6 & 99.3 & 99.3 & 8 \\
\hline 9 & 0.4 & 0.4 & 0.4 & 0.5 & 0.4 & 0.4 & 0.4 & 0.5 & & 99.7 & 99.8 & 99.5 & 99.7 & 9 \\
\hline 10 & 0.3 & 0.3 & 0.3 & 0.3 & 0.3 & 0.3 & 0.5 & 0.3 & 0.3 & & 99.8 & 99.6 & 99.6 & 10 \\
\hline 11 & 0.3 & 0.3 & 0.3 & 0.5 & 0.3 & 0.3 & 0.5 & 0.4 & 0.2 & 0.2 & & 99.6 & 99.6 & 11 \\
\hline 12 & 0.5 & 0.5 & 0.5 & 0.6 & 0.6 & 0.5 & 0.6 & 0.7 & 0.5 & 0.4 & 0.4 & & 99.4 & 12 \\
\hline \multirow[t]{2}{*}{13} & 0.4 & 0.4 & 0.4 & 0.6 & 0.6 & 0.4 & 0.2 & 0.7 & 0.3 & 0.4 & 0.4 & 0.6 & & 13 \\
\hline & 1 & 2 & 3 & 4 & 5 & 6 & 7 & 8 & 9 & 10 & 11 & 12 & 13 & \\
\hline
\end{tabular}

FPV-XY1

FPV-XY2

FPV-XY3

FPV-XY4

FPV-XY5

FPV-XY6

FPV-CU4

FPV-G

FPV-GB

FPV-HH6

FPV-IZSSI

FPV-M9132

FPV-Raccoon

Figure 1. VP2 gene homology analysis.

3) VP2 gene genetic evolution analysis:

The VP2 gene nucleotide sequence of the 6 FPV strains obtained by sequencing and the VP2 reference sequence of the selected FPV were analyzed by a phylogenetic tree (see Figure 2). The results showed that FPV-XY1, FPV-XY6, FPV$\mathrm{XY} 3$ and FPV-XY2 are closely related to each other and are on the same branch; they are closest to the reference strain FPV-RACCOON and the standard strain FPV-CU4. The relationship between the test strains FPV-XY4 and FPV-XY5 is relatively close, and their relationship with the reference strains FPV-G and FPV-HH6 is relatively close, and the relationship with other test strains is relatively distant.

4) Analysis of VP2 gene main amino acid variation analysis:

The VP2 nucleotide sequence comparison between the test strain FPV-XY1-6 and the standard strain FPV-CU4 is shown in Table 3. The VP2 gene is relatively stable, only the four test strains FPV-XY1, FPV-XY2, FPV-XY3 and FPV-XY6 at the 91st position changed from $A$ to $S$ compared to the standard strain FPV-CU4; the 101st FPV-1-6 Mutation from I to T, and the 80th (K), $93(\mathrm{~K}), 103(\mathrm{~V})$ and 323 (D) four functional sites that determine the host specificity and antigenicity are not mutated, which determines the virus. There is no mutation at position $564(\mathrm{~N})$ of hemagglutination. The VP2 gene of FPV-XY1 lacks a T at position 413 in the nucleotide sequence, which leads to an early terminator in the amino acid sequence, but there is no change in key positions.

\section{Discussion}

In recent years, the rapid development of molecular biology technology has allowed veterinarians to detect the cause of diseases at the genetic level, making the diagnosis results of diseases more accurate and convincing. In this study, 6 
stool samples of cats infected with FPV after immunization were selected to isolate the virus disease, and the virus VP2 gene was amplified, TA cloned, sequenced, and the genes were spliced to obtain the VP2 gene sequence of FPV. And the sequence homology comparison, evolution analysis, main functional site mutation analysis, found that the difference between the test strain FPV-XY1-6 and the reference strain is small, more than $99.3 \%$, the reference strains are from China in the 6 countries, this shows that the VP2 gene of FPV is relatively stable, and the mutations are relatively small in many countries around the world. The homology between the test strain FPV-XY1-6 and the standard strain FPV-CU4 is between $99.3 \%$ and $99.5 \%$. Although the homology is not as high as other reference strains, it also exceeds $99 \%$, which is in line with the current reports. The research results are consistent with [4].

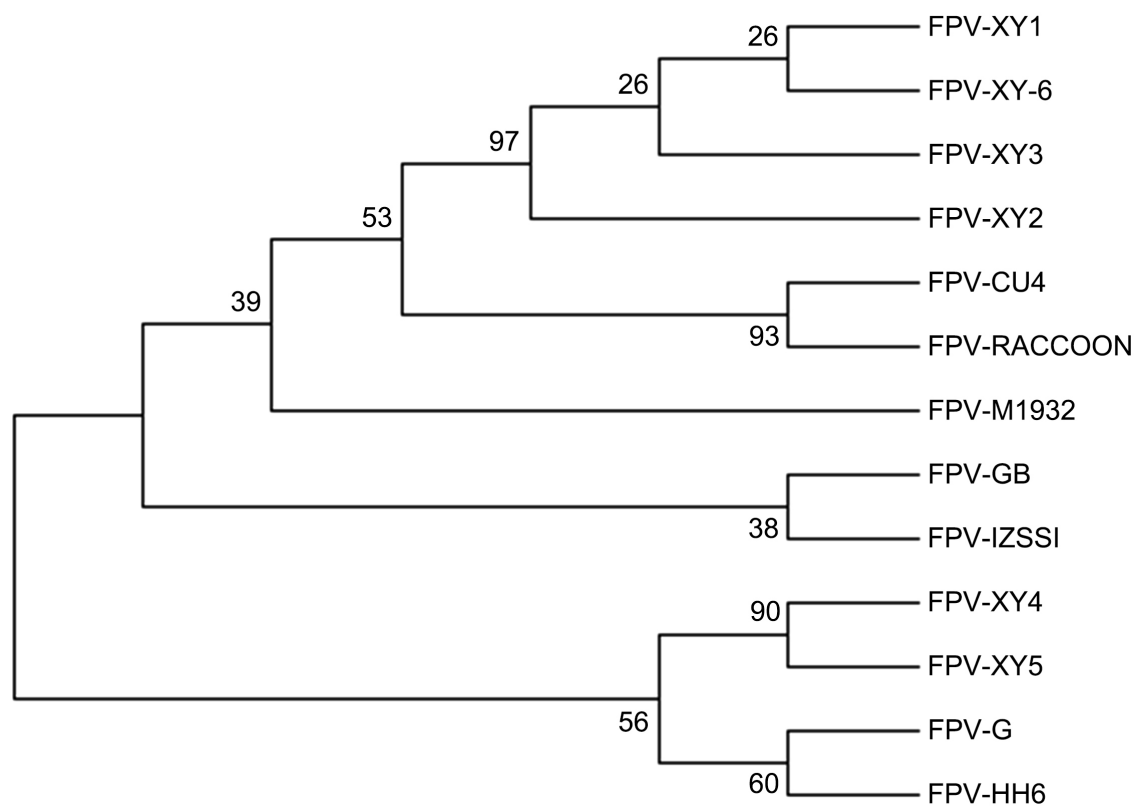

Figure 2. VP2 genetic evolution analysis.

Table 3. Primary amino acid loci VP2 genes.

\begin{tabular}{lccccccc}
\hline & \multicolumn{7}{c}{ Amino acid site } \\
\cline { 2 - 7 } Virus strain & 80 & 91 & 93 & 101 & 103 & 323 & 564 \\
\hline FPV-CU4 & $\mathrm{K}$ & $\mathrm{A}$ & $\mathrm{K}$ & $\mathrm{I}$ & $\mathrm{V}$ & $\mathrm{D}$ & $\mathrm{N}$ \\
FPV-XY1 & $\mathrm{K}$ & $\mathrm{S}$ & $\mathrm{K}$ & $\mathrm{T}$ & $\mathrm{V}$ & $\mathrm{D}$ & $\mathrm{N}$ \\
FPV-XY2 & $\mathrm{K}$ & $\mathrm{S}$ & $\mathrm{K}$ & $\mathrm{T}$ & $\mathrm{V}$ & $\mathrm{D}$ & $\mathrm{N}$ \\
FPV-XY3 & $\mathrm{K}$ & $\mathrm{S}$ & $\mathrm{K}$ & $\mathrm{T}$ & $\mathrm{V}$ & $\mathrm{D}$ & $\mathrm{N}$ \\
FPV-XY4 & $\mathrm{K}$ & $\mathrm{A}$ & $\mathrm{K}$ & $\mathrm{T}$ & $\mathrm{V}$ & $\mathrm{D}$ & $\mathrm{N}$ \\
FPV-XY5 & $\mathrm{K}$ & $\mathrm{A}$ & $\mathrm{K}$ & $\mathrm{T}$ & $\mathrm{V}$ & $\mathrm{D}$ & $\mathrm{N}$ \\
FPV-XY6 & $\mathrm{K}$ & $\mathrm{S}$ & $\mathrm{K}$ & $\mathrm{T}$ & $\mathrm{V}$ & $\mathrm{D}$ & $\mathrm{N}$ \\
\hline
\end{tabular}


The VP2 protein is the most stable protein of FPV. Compared with the standard strain FPV-CU4, the amino acid at position 101 of the VP2 protein of the tested strain FPV-XY1-6 isolated in this experiment has changed from I to T, LOOP1 of the VP2 protein. The segment is mainly composed of the 50 - 100th amino acid residues, LOOP2 is mainly composed of the 200 - 250th amino acid residues, and the 101st amino acid is next to the LOOP1 segment, between LOOP1 and LOOP2, which is similar to the LOOP1 segment reported in the literature. It is consistent with some mutations between LOOP2; in addition, this mutation may increase the virulence of the virus strain, leading to immune failure, and the related relationship needs further study. Compared with the standard strain FPV-CU4, the test strains FPV-XY1, FPV-XY2, FPV-XY3 and FPV-XY6 changed from $A$ to $S$ at the 91 st position, while the test strains FPV-XY4 and FPV-XY5. It is the same as the standard strain FPV-4, and the amino acid at position 91 is located inside the LOOP1 segment, which is inconsistent with the literature reported that it is difficult to mutate in the LOOP1 segment [5]; test strains FPV-XY1, FPV-XY2, FPV-The case corresponding to XY3 died, and only the FPV-XY6 case was cured. This may be related to the increased virulence of the strain caused by the 91st amino acid mutation; it can also indicate that if the 91 amino acid type is classified, it is likely to be prevalent in Jinan. Two FPV strains, one is an alanine strain and the other is a serine strain. The pathogenicity and virulence of the two strains need to be further proved.

In recent years, the number of pet cats raised has shown a rapid increase, which has created favorable conditions for the spread of FPV. At present, the main measures are mainly prevention. However, immune failure often occurs in preventive immunization. There are many reasons for immune failures, such as human factors, environmental factors, individual differences, and vaccine product factors. From the perspective of the vaccine itself, the current prevention of FPV in China is mainly based on the injection of inactivated FPV vaccines. Inactivated vaccines are safe to use, have less impact on maternal antibodies, and have no risk of transmission. They also have a slower onset of immunity. The immune period is short and requires multiple immunizations, which mainly stimulates the shortcomings of humoral immunity, which makes the immune failure; there is also a major factor in the mutation of the infected strain, which makes the virus strain stronger and can break through the body's immune system. Or the mutation changes the target site of the virus, so that the antibodies produced by the vaccine immunization cannot react to the mutant strain, causing immune failure. The standard strain FPV-CU4 is now used as the vaccine strain of Miao Sanduo vaccine. Miao Sanduo vaccine is currently the only cat vaccine legally operated in the Chinese market. According to statistics, its market share accounts for more than 98\%. In addition, FPV monoclonal antibodies currently on the market are all developed with reference to standard strains. This experiment proves that the FPV strains and standard strains currently circulating in Jinan have not mutated at the key sites that have been reported, and there 
are mutations in the 91 st position within the LOOP1 segment and the 101st position close to the LOOP1 segment. Studies have shown that the change of amino acid at position 101 may cause immune failure, and the change of amino acid at position 91 may increase the virulence of the strain. But this only represents the results of this test. The specific amino acid mutation at position 91 will increase the virulence of the virus, whether it will reduce the efficacy of monoclonal antibodies, and whether the amino acid mutation at position 101 will really cause some viruses to evade the immune program, leading to immunization failures require a large number of clinical cases for further verification.

From a large number of case investigations and antibody tests, it is found that most of the cats immunized with regular FPV immunization programs will produce higher antibodies, which can effectively reduce the incidence of FPV. Therefore, the Miao Sanduo vaccine of the United States can prevent FPV and play a better role. However, the problem of virus mutation cannot be ignored either. This experiment provides a certain reference for the improvement and development of FPV monoclonal antibodies and subunit vaccines.

\section{Acknowledgements}

This work was supported by a grant from the National Key Research and Development Program of China awarded to YKP(Grant No. 2017YFD0501004) and Special Economic Animal Innovation Team of Shandong Modern Agricultural Industrial Technology System (SDAIT-21).

\section{Conflicts of Interest}

The authors declare no conflicts of interest regarding the publication of this paper.

\section{References}

[1] Ikeda, Y., Mochizuki, M., Naito, R., et al. (2000) Predominance of Canine Parvovirus (CPV) in Unvaccinated Cat Populations and Emergence of New Antigenic Types of CPVs in Cats. Virology, 278, 13-19. https://doi.org/10.1006/viro.2000.0653

[2] Li, S. (2008) Isolation and Identification of Cat Parvovirus, Genome Sequence Analysis and Prokaryotic Expression of VP2 Gene. Jilin University, Jilin.

[3] Parrish, C.R., Aquadro, C.F., Strassheim, M.L., et al. (1991) Rapid Antigenic-Type Replacement and DNA Sequence Evolution of Canine Parvovirus. Journal of Virology, 65, 6544-6552. https://doi.org/10.1128/JVI.65.12.6544-6552.1991

[4] Clegg, S.R., Coyne, K.P., Dawson, S., et al. (2012) Canine Parvovirus in Asymptomatic Feline Carriers. Veterinary Microbiology, 157, 78-85.

https://doi.org/10.1016/j.vetmic.2011.12.024

[5] Parker, J.S. and Parrish, C.R. (1997) Canine Parvovirus Host Range Is Determined by the Specific Conformation of an Additional Region of the Capsid. Journal of Virology, 71, 9214-9222. https://doi.org/10.1128/JVI.71.12.9214-9222.1997 